\title{
Analysis of Synaptic Distribution within Single Retinal Axonal Arbors after Chronic NMDA Treatment
}

\author{
Laising Yen, Jane T. Sibley, and Martha Constantine-Paton \\ Department of Biology, Yale University, New Haven, Connecticut 06520
}

\begin{abstract}
Activation of the NMDA subtype of glutamate receptor has been implicated in structural synaptic plasticity in many developing sensory systems. In the frog retinotectal system, chronic exposure of the optic tectum to NMDA, which decreases the effectiveness of NMDA receptors (Debski et al., 1991), results in the pruning of the branches of retinal terminal arbors (Cline and Constantine-Paton, 1990). However, it is difficult from these studies to relate the involvement of NMDA receptors to changes in synapse distribution. In this study, we have developed an EM sampling procedure to quantitatively compare the amount and the distribution of synaptic contact within single retinal arbors. We report that within each retinal arbor, synaptic contact gradually increases from the main branches to the end branches of the arbor. Chronic NMDA treatment, however, significantly reduces the total amount of synaptic contact within each arbor. This reduced synaptic contact appears to be due to the pruning of the end branches, and the synapses these branches bear. The results are consistent with the hypothesis that NMDA receptor is an integral part of the mechanism that stabilizes coactive synapses, and that maintenance of an axonal branch requires a minimum density of synapses that are correlated with converging neighbors.
\end{abstract}

[Key words: NMDA receptor, retinotectal projection, correlated activity, three-eyed frogs, synaptic size, synaptic distribution, quantitative EM]

A variant of the Hebbian rule for cellular learning has been implicated in an activity-dependent structural changes known to occur during development. Coactivate afferents, when they cooperate to depolarize common postsynaptic partners, are postulated to reinforce or stabilize each other while inputs unable to evoke such a depolarization are destabilized and lost (Stent, 1973; Changeux and Danchin, 1976). Activation of the NMDA receptor, a subtype of excitatory amino acid receptor, has been suggested as a cellular mechanism underlying the recognition of afferent coactivity. An increasing number of reports from a variety of developing sensory systems are consistent with this idea.

\footnotetext{
Received Sept. 6, 1994; revised Dec. 2, 1994; accepted Dec. 21, 1994.

We thank Dr. Doug Kankel, Dr. Haig Keshishian, and Dr. Thom Hughes for critical reviews of the manuscript, and we are grateful to Dr. M. Mooseker for allowing us to use his EM facility and to Dr. K-Y Wei for allowing use of his morphometric measurement system. This work was supported by NIH Grant EY08760 and EY06039.

Correspondence should be addressed to Dr. Martha Constantine-Paton, Department of Biology, P.O. Box 208103, Yale University, New Haven, CT 06520-8103.

Copyright $\mathbb{C} 1995$ Society for Neuroscience $0270-6474 / 95 / 154712-14 \$ 05.00 / 0$
}

Within the central visual pathway, studies using amphibians, fish, and mammals, have shown that chronic treatments with NMDA receptor antagonists alter the structural or functional organization of retinally driven inputs (Clinc ct al., 1987; Klcischmidt et al., 1987; Bear et al., 1990; Schmidt, 1990; Udin and Scherer, 1990; Hahm et al, 1991; Simon et al., 1992). In developing cerebellum, the postnatal elimination of functionally defined multiple inputs to Purkinje cells is blocked by postnatal chronic AP5 treatment (Rabacchi et al., 1992). However the interpretation of some of these studies is ambiguous since blocking NMDA receptor activation frequently blocks activation of the cells (Shatz, 1990). Consequently, specific effects of NMDA receptor antagonism are difficult to separate from a retardation of neuropil differentiation due to a general lack of activity (Riccio and Matthews, 1985a,b; Kalil et al., 1986).

Studies using chronic application of NMDA to frog tecta have suggested an alternative approach to this issue. This treatment produces a decrease in the response of tectal neurons to NMDA without a corresponding change in the response to the nonNMDA glutamate receptor agonist AMPA (Debski et al., 1991; Hickmott and Constantine-Paton, 1991, 1993a,b, unpublished observations). In experimentally produced striped tecta, the same treatment causes a reduction in the end branches of the retinal arbors, and a removal of retinal arbor branches from the borders of eye-specific stripes (Cline and Constantine-Paton, 1990). Both the distal regions of the retinal arbors and the stripe border regions are zones of neuropil where the activity correlation among converging afferents is likely to be lower. Furthermore, the branch reduction of retinal arbors produced by chronic NMDA treatment is not observed in normal frogs where the tecta are innervated by only one eye and the activity correlation among converging afferents is likely higher. Thus, we have suggested that the observed branch reduction represents a loss of synapses from zones where their low activity correlations are no longer sufficient to promote stabilization due to the decreased responsiveness of the NMDA receptor (Cline and Constantine-Paton, 1990). Such selective pruning of synapses is expected if activation of NMDA receptors is an integral part of the mechanism that recognizes and stabilizes correlated synapses: decreases in NMDA receptor function should raise the threshold for detecting correlated inputs, and poorly correlated inputs should now be selectively eliminated. Branch reduction observed at the light microscope level, however, does not necessarily correspond to a loss of synaptic contact. Consequently, we undertook the present electron microscope study to test the hypothesis that synaptic contact of retinal axons was actually being reduced in NMDA-treated tecta. This reporl focuses on one type of RGC arbor and uses a sampling procedure to quan- 
titatively compare the amount and the distribution of synaptic contact within single retinal arbors. Our results demonstrate a significant loss of synaptic contact made by retinal arbors treated chronically with NMDA. The observations constitute the first documentation, in the CNS, of a change in synapse distribution that is associated with a decrease in the function of a particular receptor subtype.

A description of the light microscopic morphology of 2 of the retinal arbors in this article (see Fig. $6 c, h$ ) has appeared in a previous report (Yen et al., 1993).

\section{Materials and Methods}

Animals. The postmetamorphic frogs used in these studies were offspring of adult wild caught Rana pipiens purchased from Hazen and Sons, Alburg, VT. Embryos were obtained by induced ovulation and artificial fertilization in the laboratory using standard techniques. The embryonic microsurgery used to produce supernumerary eye has been detailed in previous publications (Law and Constantine-Paton, 1981). Tadpoles were fed boiled romaine lettuce. When the front legs emerged the metamorphosing tadpoles were placed in a shallow tilted container to prevent drowning. Postmetamorphic froglets were fed liberally on Drosophila melanogaster.

Chronic treatment. NMDA in concentrations that have previously been shown to prune developing retinal ganglion cell arbors without producing degenerative damage to the arbors or loss of retinal or tectal neurons (Cline and Constantine-Paton, 1990), was applied to tecta of Taylor and Kollros (1946) stage XXIV-XXV froglets. The infiltration of Elvax slabs (a gift of Du Pont) with NMDA was accomplished by adding $10 \mu \mathrm{l}$ of $10^{-2} \mathrm{M}$ NMDA solution in distilled water along with $10 \mu \mathrm{l}$ of $2 \%$ fast green in DMSO to $1 \mathrm{ml}$ of the Elvax solution (100 $\mathrm{mg}$ of Elvax dissolved in $1 \mathrm{ml}$ of methylene chloride). The solution was then frozen and cold-vacuumed to remove the organic solvent (Silberstein and Daniel, 1982). Thin slices of Elvax approximately $1000 \times$ $1000 \times 60 \mu \mathrm{m}$ were cut on a cryostat. Based on previous measurements (Cline and Constantine-Paton, 1989), we have calculated that the amount of Elvax slices implanted on each animal should release molecules in the molecular weight range of NMDA at a rate of approximately $0.005 \mathrm{nmol} / \mathrm{d}$.

Animals were anesthetized for surgery by submersion in $0.05 \%$ 3 -aminobenzoic acid ethyl ester (MS-222, Sigma). The skin, cartilaginous skull, dura and arachnoid membranes overlying the dorsal surface of the tectal lobes were retracted and the Elvax slices containing NMDA were positioned to completely cover the dorsal and lateral surfaces of both tectal lobes. The dura and cartilage were then replaced and the skin was sealed with Histoacryl glue (Tri-Hawk, Montreal). Animals were allowed to survive the treatment for 4-5 weeks. For sham-treated control animals, Elvax lacking NMDA but otherwise prepared and applied in the same way was used.

$H R P$ labeling and processing of tissue for EM. A few retinal ganglion cell (RGC) terminals arising from the ventral region of the eye of all animals were labeled by placing a pellet of the enzyme horseradish pcroxidase (HRP) in that region of the retina. This was accomplished by coating the tip of a fine insect pin with a saturated solution of HRP, and inserting the dried pin into the retina of an anesthetized animal. The injection spots were close to the peripheral retina to avoid labeling many RGC axons at once. In three-eyed animals, HRP was only placed in the supernumerary eye. We know from previous studies on threeeyed frogs that the contralateral host eye always fully innervates striped tecta (Constantine-Paton and Law, 1978; Law and Constantine-Paton, 1981), and it is the additional projection from the third eye that defines a tectal region as "doubly-innervated" (Yen et al., 1993). Thus, labeling only the supernumerary eye assured that all analyses of HRP-filled RGC axons in these animals were conducted in doubly innervated regions of tecta. The procedure eliminated the need for an additional anterograde labeling of the entire supernumerary projection with some other tracers to independently identify the region of analysis as "doubly-innervated."

Two days after the HRP-labeling the animals were anesthetized and killed by brain removal. Their tecta were fixed in $0.1 \mathrm{M}$ cacodylate buffer containing $2.5 \%$ glutaraldehyde and $1 \%$ paraformaldehyde overnight at $4^{\circ} \mathrm{C}$. The tecta were then rinsed in PBS $(\mathrm{pH} \mathrm{7.4)}$ at room temperature for $20 \mathrm{~min}$, and embedded in $37^{\circ} \mathrm{C}$ agarose solution $(3.6 \%$ w/v in phosphate buffer saline). Forty micrometer thick sections were cut parallel to the dorsal surface of tecta using a vibratome. These tissue sections were transferred to microbeakers containing cold $0.05 \mathrm{M}$ Tris saline ( $\mathrm{pH} 7.6$, with $7.8 \mathrm{gm} \mathrm{NaCl/liter)} \mathrm{in} \mathrm{sequential} \mathrm{order.} \mathrm{They} \mathrm{were}$ reacted with diaminobenzidine (DAB from Sigma), dehydrated, postfixed in $0.1 \mathrm{M}$ cacodylate buffer containing $1 \%$ osmium tetroxide and $1.5 \%$ potassium ferricyanide, and then embedded in Spurr's solution on glass slides coated with liquid releasing agent (EM Sciences). The slides were subsequently cured at $70^{\circ} \mathrm{C}$ overnight.

Axon terminal reconstruction. The morphology of isolated HRP-labeled type IV RGC (Stirling and Merrill, 1987) axons in the caudalmedial region of doubly innervated tecta were reconstructed from several of these embedded sections using a $63 \times$ oil immersion lens and a camera lucida drawing tube. Because the fixation by osmium tetroxide darkens the tectal tissue significantly, it makes visualizing HRP reaction product more difficult. Only in well-filled axons can the thinnest distal branches be visualized under the light microscope. However in these well-filled axons, many growth cones could be delineated by the HRP reaction product. Visualization of growth cones on many distal branches thereby provided a means of assuring complete filling of the axonal tree. Since different regions of tectum are slightly different in the degree of maturation during development, to ensure consistency, we attempted to labcl only ventral-nasal retina and the selection of filled RGC axons was confined to only those that fell well within the caudal-medial region of tecta. This caudal-medial region was also the region of the tectal lobe where it was easy to verify that the Elvax had remained in contact with the tectum throughout the exposure period.

This work required animals that survived at least three and up to four experimental procedures and had filled RGC axons that met rigid anatomical criteria. Consequently, more then 120 three-eyed tadpoles were produced and raised to late tadpole stages for this study. Of these, 31 three-eyed animals successfully completed metamorphosis, had a third eye that appeared healthy, recovered from the surgery for Elvax implantation, and survived the chronic treatment period and the subsequent HRP labeling. Thirteen of these animals were treated with Elvax containing NMDA, and 18 were treated with plain Elvax as controls. In addition, as a basis of comparison for RGC axonal morphology and synaptic distribution made by normal retinal axons, more than 10 stagematched, normal two-eyed animals, without Elvax treatment also had ventral-nasal retinal axons labeled with HRP.

As in previous studies using three-eyed frogs, in some of the animals that survived the experimental procedures, the supernumerary optic nerve axons failed to project to the tecta. Furthermore, we focused on only one type of RGC terminal (type IV, see below), and there is a low success rate in filling well-isolated single RGC axons to an extent that will allow complete reconstruction. Thus only a few animals had wellfilled and well-isolated type IV terminals. All quantitative analyses in this report are based on four type IV RGC axons from two NMDAtreated three-eyed animals, two type IV axons from two Control threeeyed animals, and two type IV axons from two stage-matched Normal animals. All of these axonal terminals were completely filled with good tissue preservation, and they were completely reconstructed at the light microscope level before EM measurements were begun

The Strahler ordering scheme and its modified nomenclature. The morphology of reconstructed RGC terminals was categorized using the Strahler ordering method (Strahler, 1952). With this method the end branches of an RGC axonal arbor are classified as order-1. When two branches of order- 1 converge, the branch on which they converge is assigned to order 2. Thus, in general, when two branches of order " $M$ " come together, the third branch giving rise to them is assigned to order "M+1." Where a branch of order "M" meets a branch of order "N," if " $N$ " exceeds "M," then the third bramch is assigned to order " $N$ " (Fig. 1a). With this method, a "branch" can be more than one segment long and can include many nodes. The Strahler scheme has the distinct advantage that it allows categorization beginning with the end branches, and a systematic progression back through the axonal tree to the major axon cylinder. However, the Strahler scheme always assigns the highest order to the major axon cylinder closest to the soma (order 6 in the case shown in Fig. 1a) and the lowest order (order 1) to the end branches: a convention that is opposite to that generally used in neurobiology. Furthermore, the Strahler's method will not assign the major axon cylinder to the same hierarchical level when two axons differ in their arbor complexity. Consequently, to facilitate the comparison of morphology between axons, the data on "branch order" was converted, after the ordering of the arbor was complete, to an alternate nomenclature that is used exclusively in this report. With this alternate nomenclature, the highest Strahler branch order, which was invariably the major axon 
a.

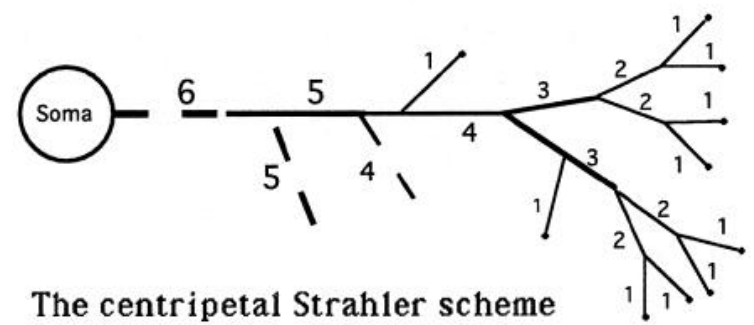

b.

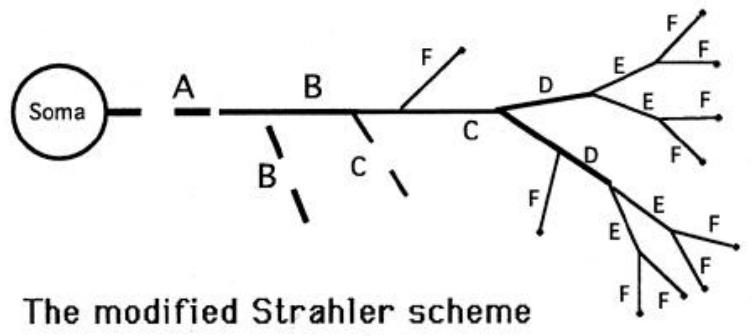

Figure 1. $a$, The Strahler ordering method. With this method the end branches of an axonal tree are classified as order-1. When two branches of order-1 converge, the branch on which they converge is assigned to order-2. Thus, in general, when two branches of order " $M$ " come together, the third branch on which they converge is assigned to order "M+1". Where a branch of order "M" meets a branch of order "N," if " $N$ " exceeds " $M$," then the third branch is assigned to order "N." $b$, The modified Strahler method. Here the highest Strahler branch order is relabeled as $A$, and branches of successively lower Strahler orders are labeled as $B, C, D \ldots$ etc. Note that those branches belonging to the same order in the original Strahler scheme will remain in the same order in the modified nomenclature.

cylinder of the ganglion cell, was labeled A, and branches of successively lower Strahler orders were labeled as B, C, D . . . etc. (Fig. 1b). Thus, the modified method assigns the major axon cylinder invariably as order A, and at the same time preserves the inner branching hierarchy of the Strahler system. Those branches belonging to the same "order" in the original Strahler scheme (Fig. 1a) will remain in the same "order" in the modified nomenclature (Fig. $1 b$ ). For example, those branches belonging to "order 2" in Figure 1 $a$ all remain in the same "order $\mathrm{E}$ " in Figure $1 b$.

Light microscopic measurement of branch length. The length of individual axonal branches in each branch order as classified by the modified Strahler scheme was measured from the light microscope reconstruction. The total length of axon in each branch order was obtained by summing individual axonal branches belonging to that branch order For this analysis, axonal branches were drawn on transparent sheets and the images were input into a Zenith Data System computer through a CCD camera (from Ikegami). The length of individual axonal branches classified by the modified Strahler scheme was then automatically measured by the morphometric software package OPTIMAS. Measurements given do not take into account the shrinkage of the tectal tissue during processing. Because the branches were measured from the dorsal projection of tectum, the depth of the arbors was not considered and the measurements were an underestimate of the actual length. This issue is treated again in the Results.

EM sampling procedure. Semithick sections (about $1 \mu \mathrm{m}$ thick) cut from embedded $40 \mu \mathrm{m}$ sections of tecta were collected on glass slides, and lightly stained with toluidine blue to visualize cell nuclei. Serial intervening ultrathin sections (about $90 \mathrm{~nm}$ thick) between these semithin sections were collected on Formvar-carbon-coated slot grids (see Fig. 2). These grids, after poststaining with uranyl acetate and lead citrate, were examined and photographed with a Zeiss-10 transmission electron microscope equipped with a goniometer. To avoid sampling the same synapse twice, nonconsecutive ultrathin sections separated by at least $0.9 \mu \mathrm{m}$ (e.g., by at least 10 ultrathin sections) were selected for analysis. Most of the HRP-labeled axonal branches reconstructed at the

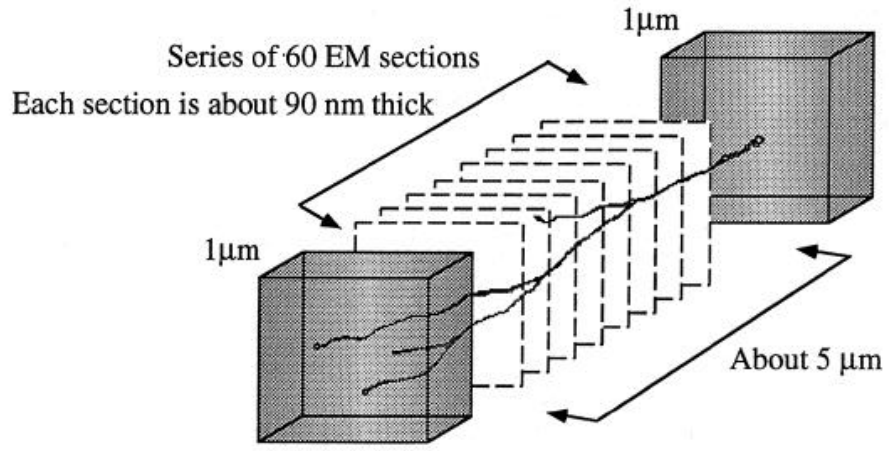

Figure 2. EM sampling method. Semithick sections (about $1 \mu \mathrm{m}$ thick) cut from embedded $40 \mu \mathrm{m}$ sections of tecta were collected on glass slides, and lightly stained with toluidine blue to visualize cell nuclei. Serial intervening ultra-thin sections (about $90 \mathrm{~nm}$ thick) between these semi-thin sections were collected on Formvar-carbon-coated slot grids. Most of the HRP-labeled axonal branches reconstructed at the light microscope level were recovered in ultrathin sections at the EM level, by using a map of cell bodies, labeled axons, and blood vessels drawn from the $1 \mu \mathrm{m}$ sections at the light microscope level.

light microscope level were recovered in ultrathin sections at the EM level, by using a map of cell bodies, labeled axons, blood vessels drawn from the semithin sections. Since these ultrathin sections cut randomly across the different parts of the HRP-labeled axons, the procedure provided a randomized sampling of synapses along the entire axonal tree. Figure $6 h$ shows an example of the distribution of branch segments that were actually sampled along the axonal tree by this randomized procedure.

Measurements of synapse size. The criteria for synapse identification was an active zone: a presynaptic density with associated synaptic vesicles and an apposed postsynaptic density. The lengths of synaptic active zones were measured by tracing the length of postsynaptic densities on transparent sheets and feeding the image of the tracing into the Zenith Data System computer through a CCD camera (from Ikegami). The length of individual active zones was then automatically measured by the morphometric software package OPTIMAS.

Estimation of branch diameter. To estimate the actual branch diameter from HRP-filled axonal profiles in EM photomicrographs, it was assumed that each axon branch was a perfect cylinder. Any section cut through a cylinder will most frequently produce an elliptical profile. The diameter $(D)$ of the cylinder can then be estimated from the individual elliptical profiles using the formula $D=4 A /(\pi \lambda)$, where $A$ is the area of the ellipse and $\lambda$ is the long length of the same profile (Fig. 3). This value $4 A /(\pi L)$ is independent of the cutting angle of the section to the cylinder. To estimate the average diameter for the entire branch order, individual $4 A /(\pi \lambda)$ values measured from HRP-filled profiles belonging to the same branch order were averaged.

Measurement of the proportion of presynaptic membrane involved in synaptic contact. For each HRP-filled axonal profile, the length of axonal membrane (designated as $L p$ ), and the length of synaptic active zone (designated as $L s$ ) were measured. The ratio $L s / L p$ thus represents the proportion of pre-synaptic axon membrane involved in synaptic contact for that HRP-filled profile. To estimate the overall " $L s / L p$ " ratio for a particular branch order, individual $L s$ and $L p$ values measured from branches belonging to the same order were summed together as $\Sigma L p$ and $\Sigma L p$, respectively (Fig. 4). The ratio $\Sigma L s / \Sigma L p$ is thus an overall estimate of the proportion of axonal membrane involved in synaptic contact for those HRP-filled branches belonging to the same branch order.

Estimation of total synaptic contact made by single retinal axons. If the length and the diameter of the branches are known, then the "total membrane area" of those branches can be estimated as " $\pi \times$ branch diameter $\times$ branch length." If the proportion of the axonal membrane involved in synaptic contact can also be measured, the total amount of synaptic contact made by these branches can then be estimated as "total membrane area $\times$ the proportion of membrane involved in synaptic contact." In other words, the formula " $\pi \times$ diameter $\times$ branch length $\times(\Sigma L s / \Sigma L p)$ " estimates the amount of synaptic contact area made by each branch order (Fig. 5). These values were added across all branch 


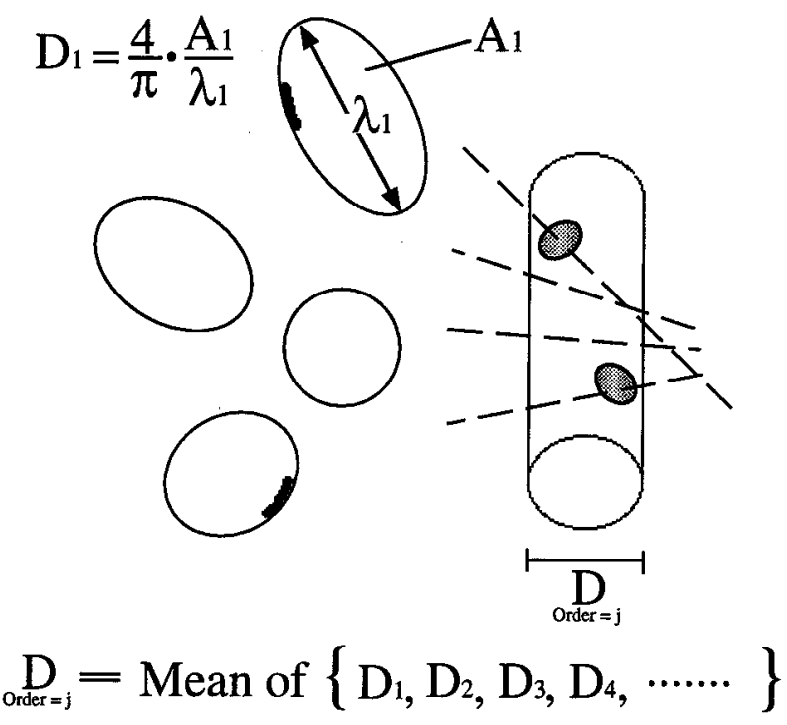

Figure 3. Measurement of branch diameter. Any section cutting through an axonal cylinder will produce an elliptical profile. The diameter $(D)$ of the axonal cylinder can be estimated from the individual elliptical profiles using the formula $D=4 A /(\pi \lambda)$, where $A$ is the area of the ellipse and $\lambda$ is the long length of the same profile. This value $4 A /(\pi L)$ is independent of the cutting angle of the section to the cylinder. To estimate the average diameter for an entire branch order, individual $4 A /(\pi L)$ values measured from HRP-filled profiles belonging to that branch order were averaged.

orders, giving a value that estimates the total synaptic contact for the entire arbor.

Estimation of axonal volume. The formula $\left[V=L \pi(D / 2)^{2}\right]$ order $=j$ estimates the axonal volume of branches of order $j$, where $L$ is the total length of branches of the order $j$, and $D$ is the estimated average diameter for the same branch order. The axonal volume of single RGC axonal arbors can be calculated from the sum of its all branch orders $[\Sigma V]$ order $=\mathrm{B}$ to $\mathrm{F}$. This axonal volume estimation includes the entire arborization but excludes the major $\mathrm{A}$ branch because it extends back to the retina and its length cannot be measured.

\section{Results}

The identification of axonal arbors from a single retinal cell type

One difference between the morphological analysis in this study and that of the previous work (Cline and Constantine-Paton, 1990 ) is that here we have concentrated the study on the type IV class of RGC axonal arbors (Grüsser and Grüsser-Cornehls, 1964). These arbors, believed to arise from the dimming detectors of retinal ganglion cells (Maturana et al., 1960; Stirling and Merrill, 1987), project deep to layer 8 of the tectum. They are large arbors extended from 300 to $500 \mu \mathrm{m}$ in the rostrocaudal direction, and myelinated along the most proximal regions of the main branches. These arbors constitute a homogeneous population that can be identified from animal to animal, and are ideally suited intense EM study.

Eight such type IV RGC axonal arbors were completely reconstructed and used for quantitative FM analyses. They were drawn from three different experimental groups of tecta: singly innervated tecta of normal two-eyed animals (Normal arbors), doubly innervated tecta, sham-treated with Elvax (Control arbors), and doubly innervated tecta treated with Elvax containing NMDA (NMDA-treated arbors). Figure $6 a-h$ shows complete light microscope reconstructions of the eight axonal arbors.

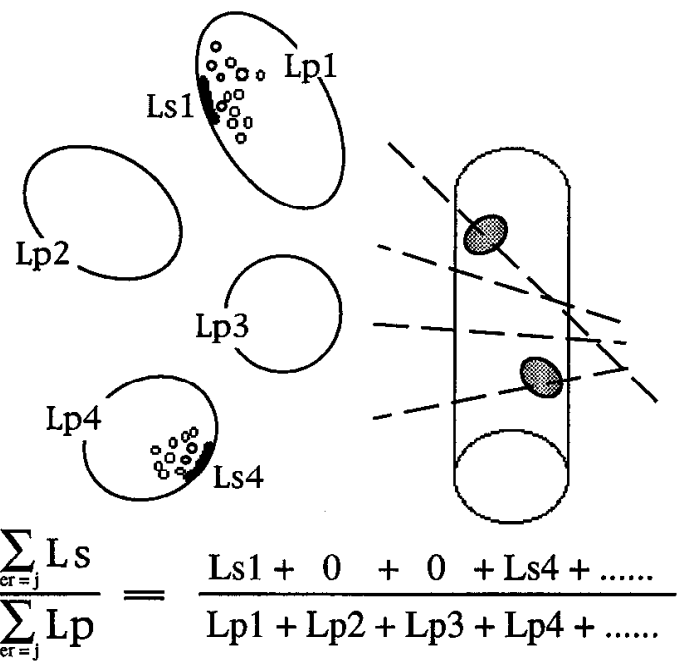

Figure 4. Measurement of the proportion of presynaptic membrane involved in synaptic contact. For each HRP-filled axonal profile, the length of axonal membrane (designated as $L p$ ), and the length of synaptic active zone (designated as $L s$ ) were measured. To estimate the overall $L s / L p$ ratio for a particular branch order, individual $L s$ and $L p$ values measured from branches belonging to the same order were summed together as $\Sigma L p$ and $\Sigma L p$, respectively. The ratio $\Sigma L s / \Sigma L p$ is thus an overall estimate of the proportion of axonal membrane involved in synaptic contact for those HRP-filled branches belonging to the same branch order.

\section{Analyses of branching morphology of the RGC arbors}

Although previously it was shown that NMDA treatment reduced the branching of RGC arbors in the superficial layers of the tectal neuropil (Cline and Constantine-Paton, 1990), it was not certain when we began this work that NMDA treatment would have the same effect on the type IV arbors located in layer 8 of the tecta. In our earlier report we showed that the type IV arbors show morphological changes that are qualitatively similar to the retinal ganglion cell arbors analyzed earlier (Yen

$$
\begin{aligned}
& \text { Total synaptic contact area of a branch } \\
& =(\pi \mathrm{DL}) \cdot(\Sigma \mathrm{Ls} / \Sigma \mathrm{L} \mathrm{p})
\end{aligned}
$$

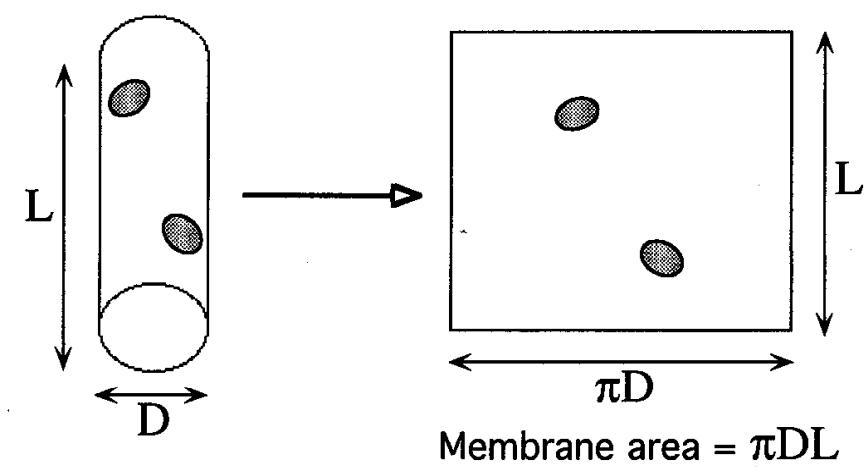

Figure 5. Estimation of total synaptic contact made by a branch. The membrane area of a branch can be estimated as $\pi D L$, where $D$ is the diameter of the branch, and $L$ is the length of the branch. The total amount of synaptic contact made by a branch can be estimated as "total membrane area $X$ the proportion of membrane involved in synaptic contact." In other words, the formula $\pi D L \times(\Sigma L s / \Sigma L p)$ estimates the amount of synaptic contact area made by a branch. 


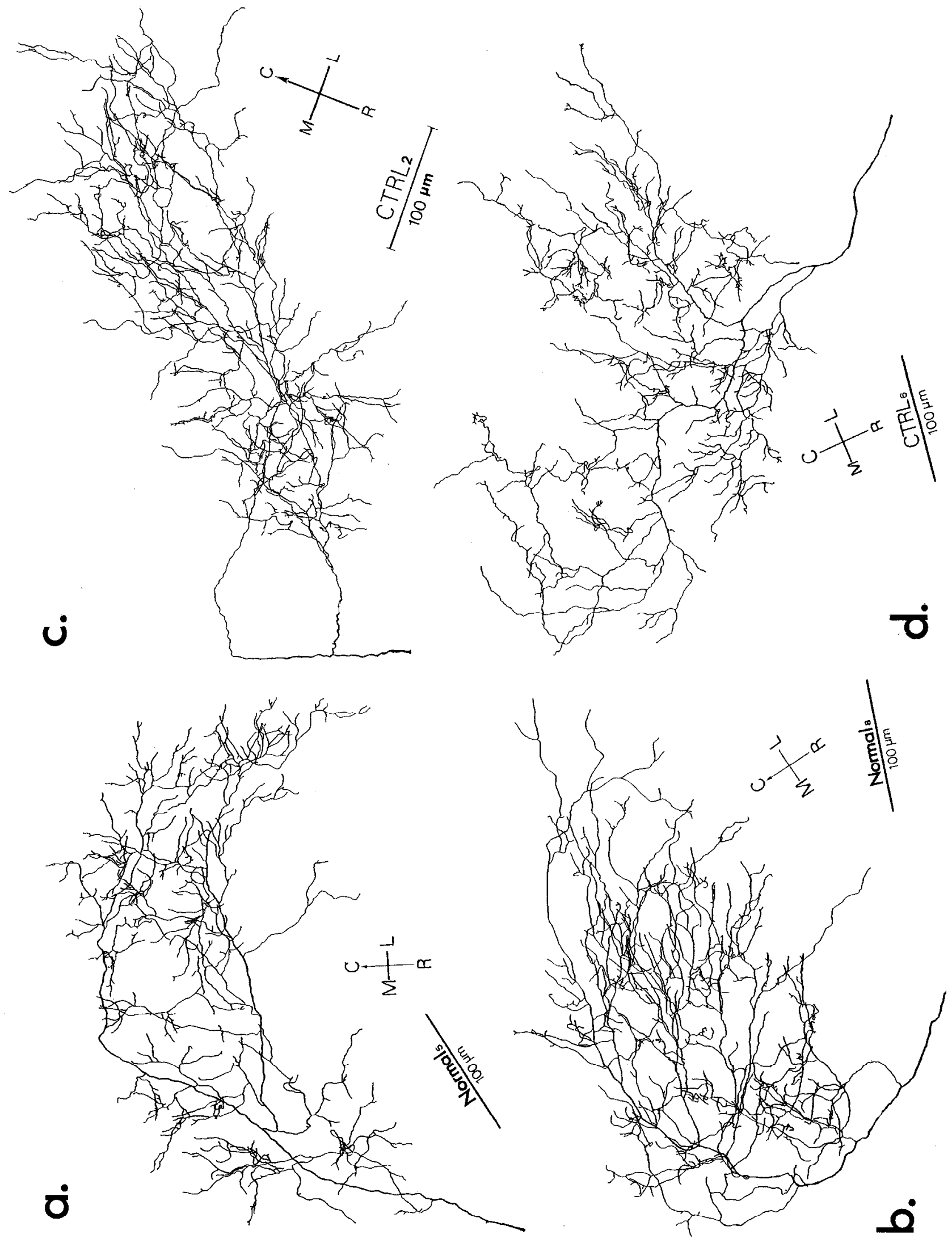



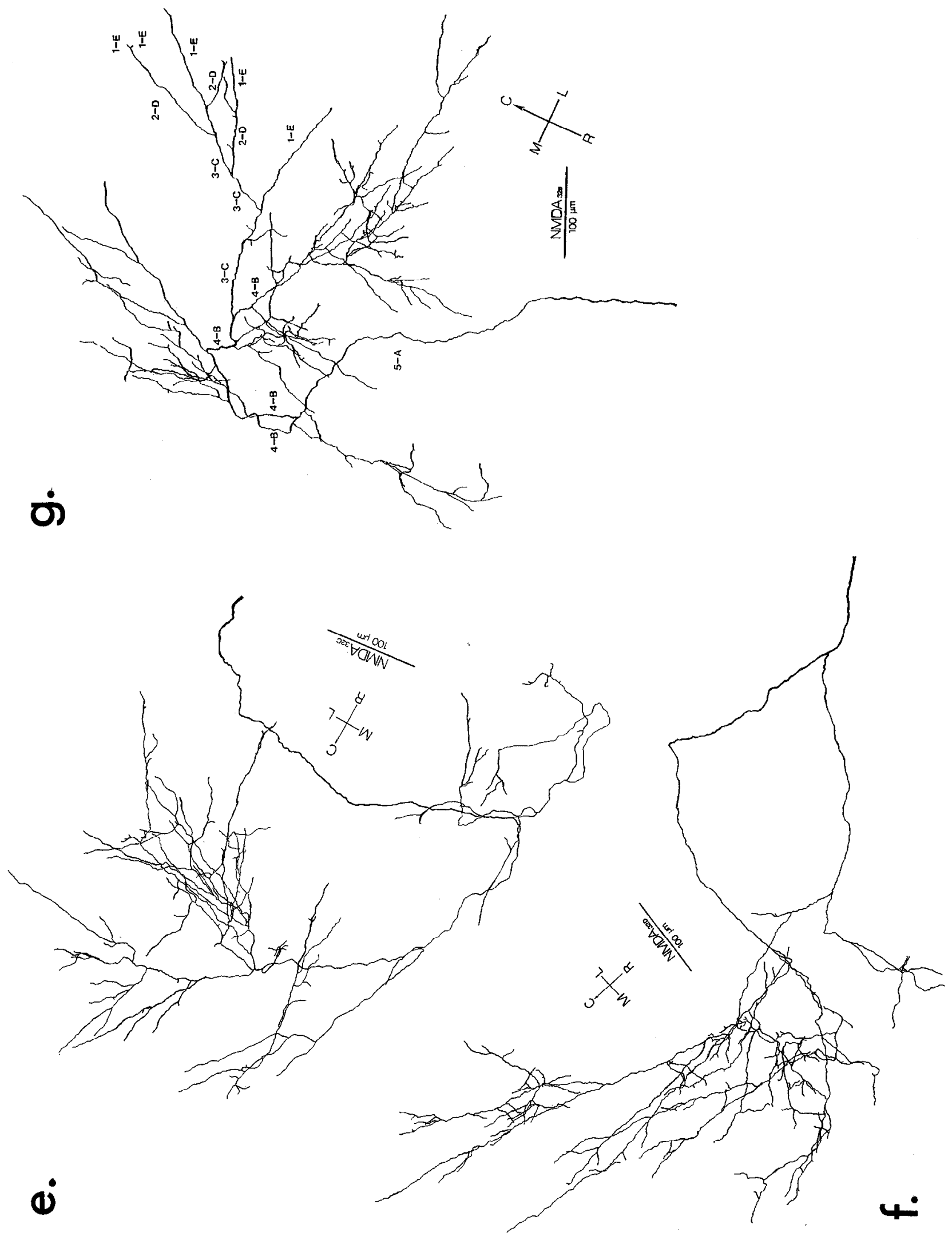


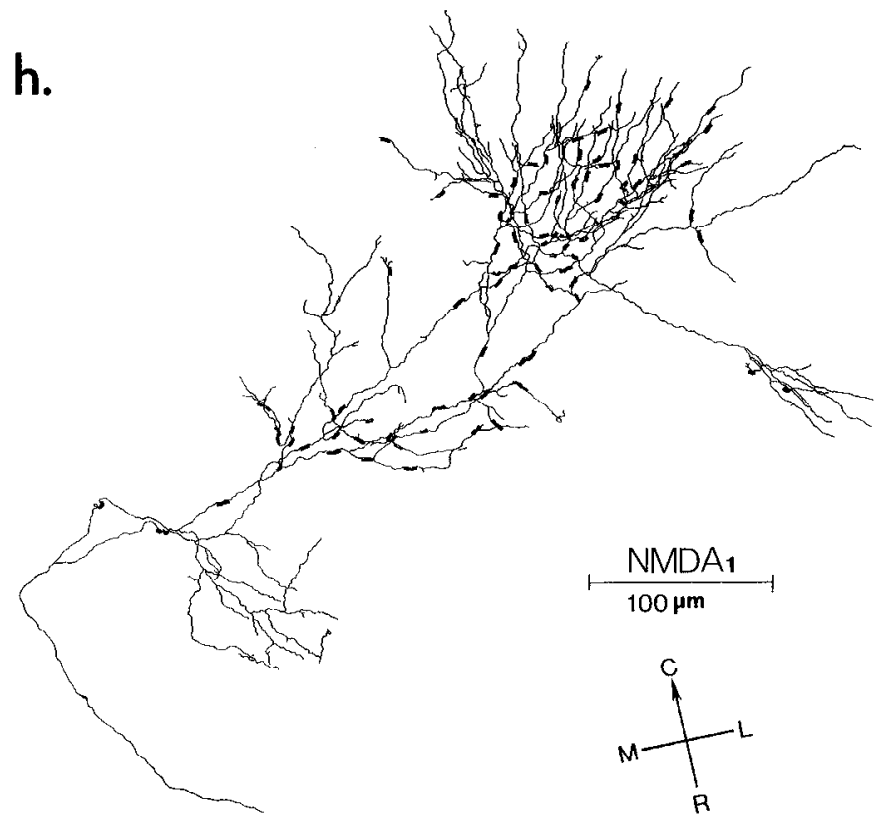

Figure 6. Complete light microscope reconstructions of the eight type IV RGC axonal arbors. $a$ and $b$ were drawn from singly-innervated tecta of normal two-eyed animals (Normal arbors). $c$ and $d$ were drawn from doubly-innervated tecta, sham-treated with Elvax (Control arbors). $e-h$ were drawn from doubly innervated tecta treated with Elvax containing NMDA (NMDA-treated arbors). Note that branching was reduced in all NMDA-treated arbors. $g$ shows an example of ordering the branches using the modified Strahler scheme. In this NMDA-treated arbor, the highest Strahler branch order reached is 5. This is then relabeled $A$, and branches of successively more distal Strahler orders are relabeled $B, C, D, E . h$ shows an example of the distribution of branch segments (the red segments) that were actually sampled using EM procedures. The positions of these segments along the axonal tree were randomly selected as a result of how they fell within the series of 90 $\mathrm{nm}$ sections lying between the two semithin sections (see Fig, 2 and Materials and Methods). $R$, rostral; $M$, medial; $L$, lateral; $C$, caudal. Scale bar, $100 \mu$ III.

$\leftarrow$

et al., 1993). In this study, the modified Strahler ordering scheme (see Materials and Methods and Fig. 1) is used to categorize the morphology of type IV axonal arbors, and provide a way to perform a quantitative EM study that can test for differences in synapse distribution across branch orders and across treatments. An example of branch ordering using both the original and the modified Strahler scheme is illustrated in Figure $6 g$. Throughout this study, we use the term "proximal branches" to refer to modified Strahler branch orders A and B, and the term "distal branches" to refer to branches of orders $E$ and $F$.

Using this method we first compared the modified Strahler description of different axons within the same treatment group. We found that the number of branches in each branch order under the modified Strahler system varied little from axon to axon (Fig. 7a,b). The number of hierarchical levels achieved by arbors in each treatment group is also very consistent (A to $\mathrm{E}$ for all NMDA-treated arbors, and A to F for all non NMDAtreated arbors). Thus, the modified Strahler system appears to accurately describe the hierarchical organization of the type IV arbor.

Similar to the results of previous studies (Cline and Constantine-Paton, 1990), the data indicate a significant reduction in

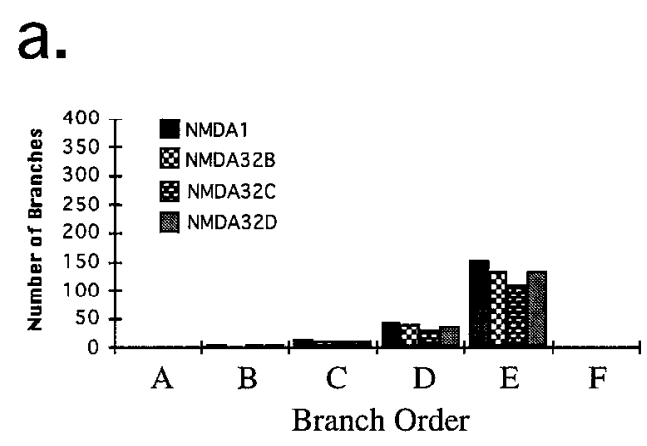

b.
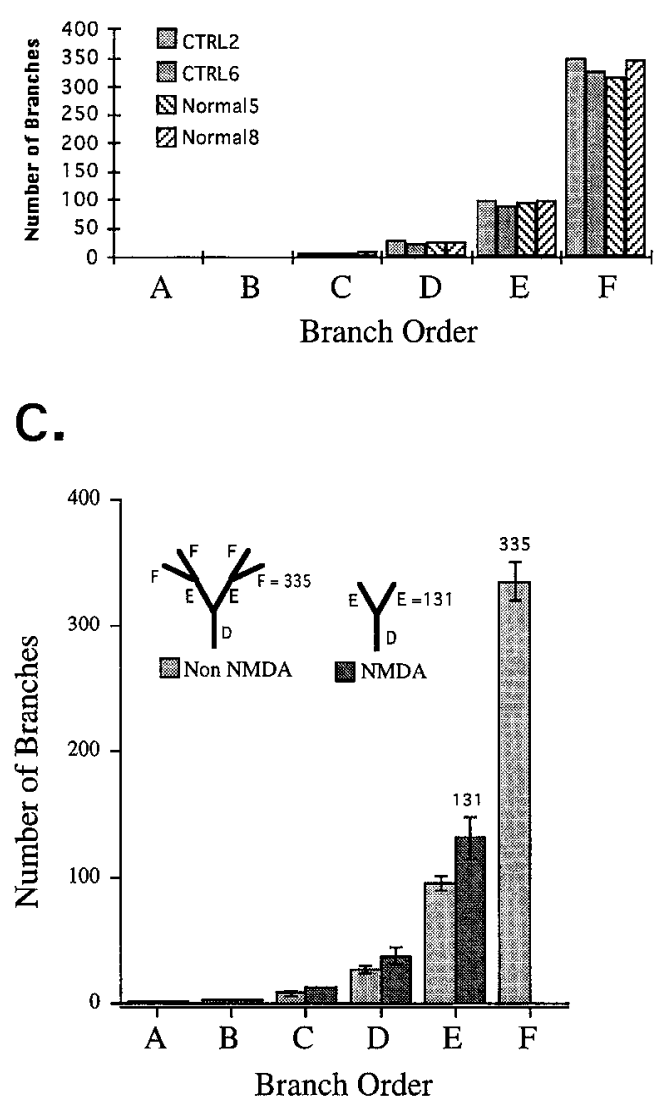

Figure 7. The number of branches in each branch order as defined by the modified Strahler scheme. $a$, The number of branches in each order of the four NMDA-treated arbors. $b$, The number of branches in each order of the two Normal arbors and two Control arbors. $c$, The average number of branches in each branch order for NMDA-treated and non NMDA-treated arbors. Data were grouped from the Control and Normal arbors to form the non-NMDA-treated group. The decreased branching in the NMDA-treated arbors is seen as a loss of the $F$ branch order, and an reduction of end branches (131 vs 335 ). The inset graphically illustrates this difference in morphology.

branching of type IV arbors following treatment of doubly-innervated tecta with NMDA. The four NMDA-treated arbors had $151,132,110,132$ end branches as compared to the 350 and 328 in Control arbors, and to the 318 and 345 in the Normal arbors. On average, this is a reduction of $60 \%$ in NMDA-treated arbors relative to the Control and Normal arbors. This analysis also shows that the NMDA-treated arbors have fewer branch orders in their hierarchy: they range from order A through order 
E whereas the Control and Normal arbors range from A through F (Fig. $7 a, b$ ).

Figure $7 b$ also provides comparisons of Normal arbors in singly innervated tecta, and Control arbors in doubly innervated tecta. In addition to the same branching hierarchy of order A through $F$, the number of branches in each order had similar values and distributions across the Normal and Control arbors. The similarities thus allowed grouping of data from these two non NMDA-treated groups. Figure $7 c$ compares the average number of branches of all non NMDA-treated arbors to that of all NMDA-treated arbors. The reduction in the number of end branches is reflected as a loss of the $\mathrm{F}$ branch order in the NMDA-treated arbors. This effect of NMDA treatment is graphically illustrated in the figure inset.

\section{EM serial reconstruction of branch segments}

Figure 8 shows representative profiles of HRP-filled axons in regions where the axon makes synaptic contact with a postsynaptic process. The figure illustrates that we were able to distinguish synaptic release sites across a wide range of HRP labeling intensities.

To determine quantitatively whether the loss of axon branches with NMDA treatment actually represented a loss of synapses, we developed an EM sampling procedure for estimating the amount of axon membrane in synaptic contact for each RGC axonal arbor. The procedure is described in detail in Materials and Methods. However, before relying on any sampling procedure, it was necessary to gain an understanding of the frequency and distribution of presynaplic release sites along these RGC axonal branches. Therefore, several branch segments (one of a $B$ segment, five from $E$ segments and two from $F$ segments) with lengths ranging from 2 to $28 \mu \mathrm{m}$ were reconstructed from the non NMDA-treated and NMDA-treated retinal arbors. A representative reconstructed branch is shown in Figure 9, where black represents the HRP-filled presynaptic membrane, red the synaptic active zone, and blue the postsynaptic membrane. Synapses made by these HRP-labeled branches are sparsely distributed, and there is no apparent systematic clustering of synaptic release sites. In addition, these synapses occupy only a relatively small proportion of the membrane of HRP-filled axons. We have observed a few cases of postsynaptic profiles that receive converging synapses both from the HRP-labeled retinal axons and from an unlabeled process. Occasionally, the postsynaptic profile itself makes synaptic contact with an unlabeled process, forming a scrial synapse. In these reconstructed segments, we could not distinguish any qualitative difference in synapse distribution within NMDA and non-NMDA treated arbors.

\section{Synapse sampling paradigm}

To make quantitative estimates of the amount of synaptic contact in an axonal arbor, three parameters must be measured: the length of branches, the diameter of branches, and the proportion of the membrane of those branches involved in synaptic contact (see Fig. 5 and Materials and Methods). For each RGC axonal arbor, we measured these three parameters and catalogued the data according to branch order using the modified Strahler scheme. The length of branches can be measured from the light microscope reconstructions. The diameter of branches and the proportion of the membrane of those branches involved in synaptic contact, however, must be measured at the EM level. The EM sampling procedure we developed provided a randomized sampling of axon segments along the entire axonal tree (see Fig.
2 and Materials and Methods). Figure $6 h$ shows an example of the distribution of branch segments that were actually sampled along the axonal tree by this randomized procedure.

\section{The length of axonal branches}

Lengths of axonal branches in each branch order were measured from the light microscope reconstructions beginning at the first bifurcation of the major axon cylinder. Figure 10 shows the average length of axonal branches in each Strahler order plotted as a histogram comparing the NMDA-treated and the nonNMDA-treated arbors. In both NMDA-treated and non-NMDAtreated arbors, the measured mean length of branches decreased gradually from the $\mathrm{B}$ order to the $\mathrm{E}$ or $\mathrm{F}$ order. Comparison of NMDA and non-NMDA-treated arbors revealed that the mean branch length of $\mathrm{B}, \mathrm{C}, \mathrm{D}$, and $\mathrm{E}$ orders remained unchanged (NMDA vs non NMDA, One-way ANOVA. B branches, $p=$ 0.603 ; C branches, $p=0.593$; D branches, $p=0.285$; E branches, $p=0.671$ ). However, the length of the end branches (the $\mathrm{E}$ branches) of NMDA-treated arbors were significantly longer than that of the end branches (the F branches) of non NMDAtreated arbors $(p=0.002)$. Thus, the end branches in NMDAtreated arbors are not equivalent to the end branches in nonNMDA-treated arbors. This quantitative observation is consistent with the hypothesis derived from previous observations that the reduction in the NMDA-treated axonal arbors occurs mainly by elimination of the end branches of the arbor.

The total axonal length in each branch order was calculated as the sum of the lengths of the individual branches of the same order (Table 1, top). Table 1 (bottom) presents these length measurements as a percentage of the total axonal length within the arborizations. In non-NMDA-treated arbors, the end branches (order F) contribute almost $50 \%$ of the entire arbor length in all the axons. This is also true of the end branches (order E) of NMDA-treated arbors. When total axonal length of each arbor was calculated as the sum across all branch orders, the total axonal length in NMDA-treated arbors $(5858 \mu \mathrm{m}, 7159 \mu \mathrm{m}$, $6457 \mu \mathrm{m}, 7204 \mu \mathrm{m}$ ) was always less than the total length in non-NMDA-treated arbors $(9877 \mu \mathrm{m}, 8460 \mu \mathrm{m}, 7432 \mu \mathrm{m}, 8915$ $\mu \mathrm{m})$.

\section{The diameter of axonal branches}

As shown in Figure 11, the mean diameters of the branches assigned to a particular Strahler order decreased gradually from the major axon cylinder to the end branches of the arbor. When the diameter measurements between all NMDA and all nonNMDA-treated arbors are compared, the data indicate that $\mathrm{A}, \mathrm{B}$, $C$, and D order branches in the NMDA-treated arbors remained roughly unchanged compared to that of non-NMDA-treated arbors (NMDA vs non-NMDA one-way ANOVA: A branches, $p$ $=0.287$; $\mathrm{B}$ branches, $p=0.075 ; \mathrm{C}$ branches, $p=0.107$; $\mathrm{D}$ branches, $p=0.593$ ). However, the diameter of $\mathrm{E}$ branches (the end branches) of NMDA-treated arbors are bigger than that of the $\mathrm{E}$ and $\mathrm{F}$ branches (the two most distal branch orders) of nonNMDA-treated arbors $(p-0.001$ and $p<0.001$, respectively). Thus, loss of the $\mathrm{F}$ branch order in the NMDA-treated arbors appears to be associated with an increase in the caliber of the branch order that becomes the new end branch of the arbor.

The diameter measurements, when combined with branch length data, allowed us to calculate the volume occupied by individual retinal axons. The results are shown in Figure 12. Surprisingly, the branch reduction caused by NMDA treatment does not decrease the total volume of individual arbors. It ac- 

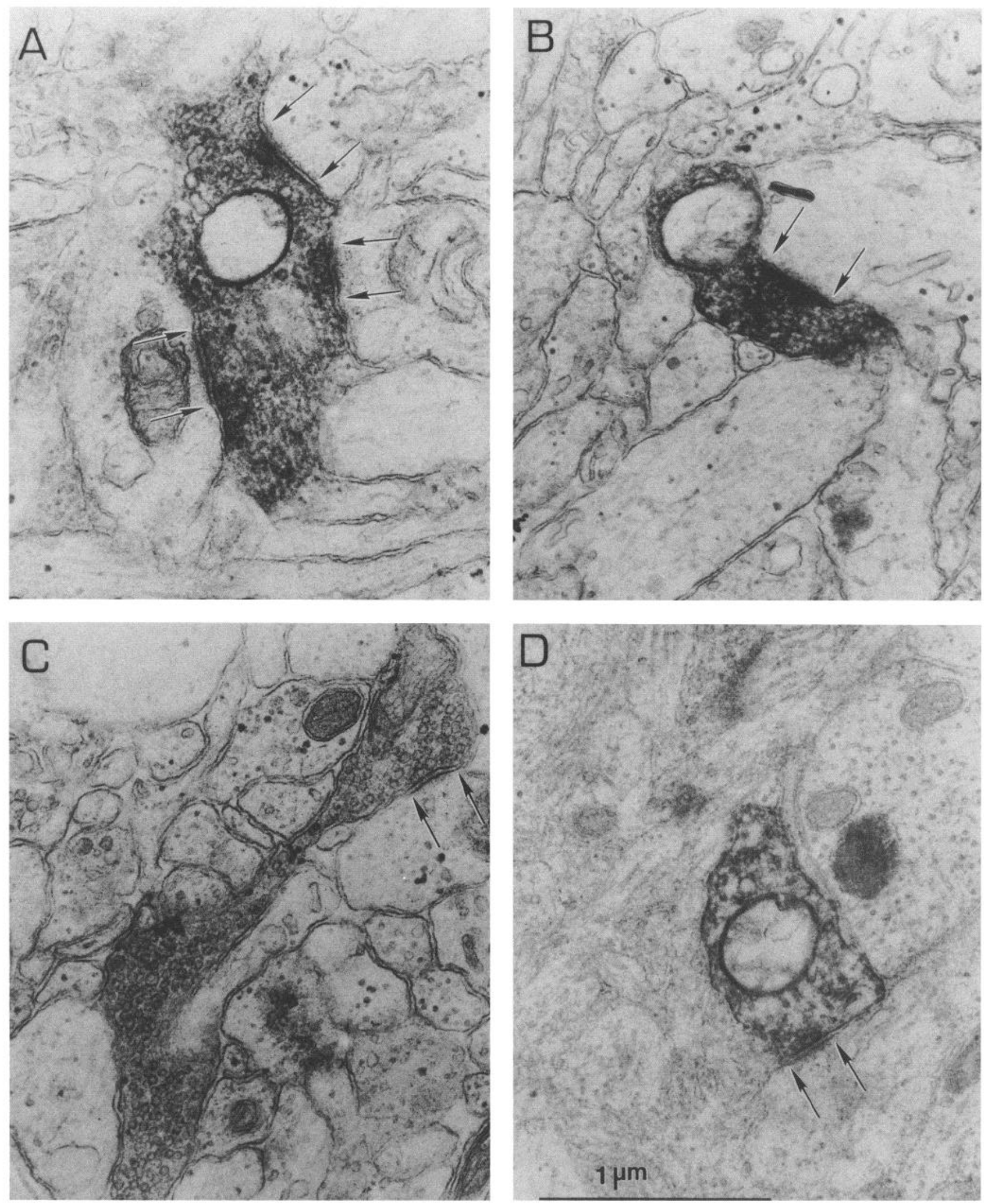

Figure 8. Examples of HRP-filled axonal profiles. Arrows point from the postsynaptic to the presynaptic site. The criteria for synapse identification was an active zone: a presynaptic density with associated synaptic vesicles and an apposed postsynaptic density. The figure illustrates that we were able to distinguish synaptic release sites across a wide range of HRP-labeling intensities. 


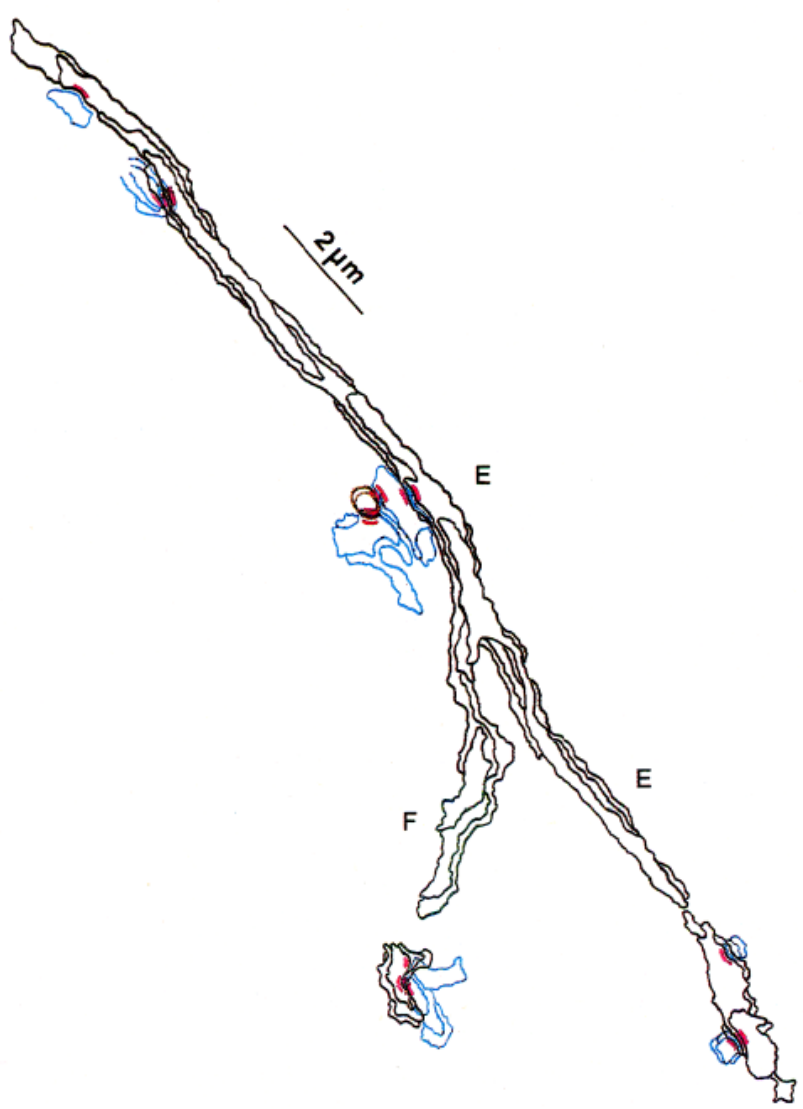

Figure 9. Representative reconstructed axonal branches from a nonNMDA-treated arbor. The reconstructed axonal branches include a partial E branch and a partial F branch. Black represents the HRP-filled presynaptic membrane, red the synaptic active zone, and blue the postsynaptic membrane. Occasionally, the postsynaptic profile (blue) itself makes synaptic contact with an unlabeled process (brown), forming a serial synapse. Synapses made by these HRP-labeled branches are sparsely distributed, and these synapses occupy only a relatively small proportion of the membrane of HRP-filled axons.

tually increases the volume of the axonal tree (one-way ANOVA, $p=0.018$ ).

\section{Estimates of the proportion of axon membrane involved in synaptic contact $(\Sigma \mathrm{Ls} / \Sigma \mathrm{Lp})$}

We estimated the proportion of the membrane involved in synaptic contact by the ratio $(\Sigma L s / \Sigma L p)$ for each branch order, as illustrated in Figure 4. Here $\Sigma L s$ is "the total length of synaptic active zones" observed in the presynaptic, HRP-filled EM profiles, and $\Sigma L p$ is "the total axon membrane length" observed in those same profiles. This ratio was calculated for each branch order of all the type IV retinal arbors, and the data are shown in Figure 13. Since myelin covers most of the A branch of type IV retinal arbors, the $\Sigma L s / \Sigma L p$ values of the A branches were not measured.

In general, branches of all orders except $\mathrm{A}$, are involved in synaptic contact. However, the $\Sigma L s / \Sigma L p$ values appear to be smaller in proximal branch orders, and larger in distal branch orders. Note that the end branches (order F) of non-NMDAtreated arbors are heavily involved in making synapses as revealed by their high $\Sigma L s / \Sigma L p$ value. Comparisons of the $\Sigma L s /$ $\Sigma L p$ values of NMDA-treated arbors to that of non-NMDAtreated arbors indicate that the B, C, D, E branches remained

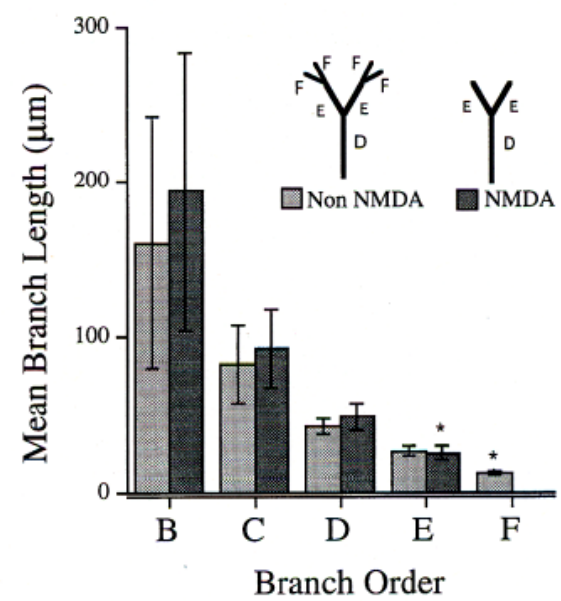

Figure 10. Mean length of branches of each Strahler order. The mean length decreased gradually from the $\mathrm{B}$ order to the $\mathrm{E}$ or $\mathrm{F}$ order in both NMDA-treated and non-NMDA-treated arbors. The lengths of the major axon cylinders (order A) were not included since they extend from the retina to the tectum, and they are myelinated and do not contain synapses. The mean branch length of B, C, D, and E orders in the NMDAtreated arbors remained unchanged compared to that of non-NMDAtreated arbors. The length of the end branches (order E) of NMDAtreated arbors are apparently longer than that of the end branches (order F) of non NMDA-treated arbors (indicated by $* ; p=0.002$ ). This effect of NMDA treatment on branch length is graphically illustrated in the inset.

roughly unchanged in this parameter (NMDA vs non-NMDA one-way ANOVA: B branches, $p=0.384$; $\mathrm{C}$ branches, $p=$ 0.106 ; D branches, $p=0.065$; E branches, $p=0.112$ ). However, for the end branches (order E) of NMDA-treated arbors, the $\Sigma L s$ / $\Sigma L p$ value is significantly lower than that of the end branches (order F) of non-NMDA-treated arbors (E vs F branches, $p<$ 0.014).

It is interesting that neither the branch length nor the $\Sigma L s /$ $\Sigma L p$ value were significantly changed in $\mathrm{B}, \mathrm{C}, \mathrm{D}$, and $\mathrm{E}$ branches with NMDA treatment. The only significant change in the NMDA-treated arbors was found in the diameter of E branches, the new end branches whose caliber became larger.

We also calculated the $\Sigma L s / \Sigma L p$ values of the several branch segments that were serially reconstructed at EM level and shown earlier in Figure 9. The $\Sigma L s / \Sigma L p$ values of these branch segments ranged from $1.1 \%$ to $3.7 \%$. This range of $\Sigma L s / \Sigma L p$ values is similar to that obtained in the sampled data of Figure 13, thus supporting the validity of the sampling methods used. The exception was one short $\mathrm{F}$ branch with no synapse. Perhaps this was a branch fixed at the moment of extension or retraction.

\section{Total synaptic contact area of single arbors}

The $\Sigma L s / \Sigma L p$ values reflect the proportion of presynaptic axon membrane involved in synaptic contact. To estimate the total amount of synaptic contact, we multiplied the "total membrane area" by " $\Sigma L s / \Sigma L p$ " for each branch order (see Fig. 5 and Materials and Methods). The estimated values of synaptic contact area contributed by branches of each order are shown in Figure $14 a$.

Comparison of NMDA-treated arbors to the non-NMDAtreated arbors shows that the synaptic contact contributed by $\mathrm{B}$, $C, D, E$ branch orders remained unchanged with NMDA treatment (one-way ANOVA: B branches, $p=0.051$; $\mathrm{C}$ branches, $p$ $=0.067 ; \mathrm{D}$ branches, $p=0.967 ; \mathrm{E}$ branches, $p=0.187)$. How- 
Table 1. Total length of axonal branches in each branch order and as a percentage of the total axonal length

\begin{tabular}{|c|c|c|c|c|c|c|c|c|}
\hline Order & Normal5 & Normal8 & CTRL2 & CTRL6 & NMDA1 & $\begin{array}{l}\text { NMDA- } \\
\text { 32B }\end{array}$ & $\begin{array}{l}\text { NMDA- } \\
32 \mathrm{C}\end{array}$ & $\begin{array}{l}\text { NMDA- } \\
\text { 32D }\end{array}$ \\
\hline \multicolumn{9}{|c|}{ Total length of branches $(\mu \mathrm{m})$} \\
\hline A & - & - & 一 & - & - & - & - & - \\
\hline B & 244.4 & 267.8 & 561.5 & 212.1 & 488.2 & 536.6 & 242.2 & 1054.8 \\
\hline $\mathrm{C}$ & 657.0 & 757.0 & 671.9 & 433.9 & 883.6 & 878.7 & 1244.7 & 1255.7 \\
\hline D & 1068.7 & 1179.0 & 1142.2 & 1143.9 & 1611.5 & 2018.4 & 1738.8 & 1764.0 \\
\hline $\mathrm{E}$ & 2117.2 & 2478.7 & 2948.7 & 2417.1 & 2874.4 & 3725.5 & 3230.9 & 3129.2 \\
\hline $\mathrm{F}$ & 3344.5 & 4232.3 & 4552.7 & 4253.3 & & & & \\
\hline Total & 7431.8 & 8914.7 & 9877.0 & 8460.4 & 5857.6 & 7159.2 & 6456.7 & 7203.7 \\
\hline \multicolumn{9}{|c|}{ Percentage of total axonal length (\%) } \\
\hline A & - & - & - & - & - & - & - & - \\
\hline B & 3 & 3 & 6 & 3 & 8 & 7 & 4 & 15 \\
\hline $\mathrm{C}$ & 9 & 8 & 7 & 5 & 15 & 12 & 19 & 17 \\
\hline $\mathrm{D}$ & 14 & 13 & 12 & 14 & 28 & 28 & 27 & 24 \\
\hline $\mathrm{E}$ & 28 & 28 & 30 & 29 & 49 & 52 & 50 & 43 \\
\hline $\mathrm{F}$ & 45 & 47 & 46 & 50 & & & & \\
\hline Total & 100 & 100 & 100 & 100 & 100 & 100 & 100 & 100 \\
\hline
\end{tabular}

ever, the end branches (order F) of the non-NMDA-treated arbors contribute almost $50 \%$ to the total synaptic contact of the arbors. Since the NMDA-treated arbors lack the F branch order and they do not increase the synaptic contact on the remaining branch orders, the total synaptic contact area of the NMDAtreated arbors is reduced relative to the non-NMDA-treated arbors.

To compare the difference in synaptic contact area between NMDA and non-NMDA-treated arbors, the total synaptic contact area made by these arbors was calculated by summing across all branch orders. Figure $14 b$ shows the averaged total synaptic contact area of all NMDA-treated and all non-NMDAtreated arbors. Estimated in this way, the reduction in synaptic

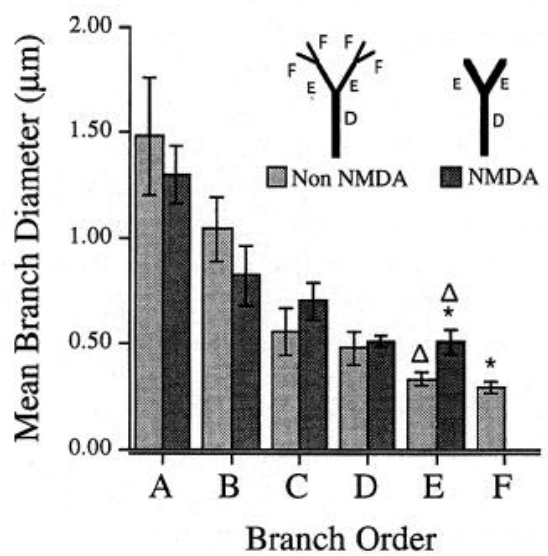

Figure 11. Mean branch diameter. The diameters of the branches decreased gradually from the major axon (A order) to the end branches in all the RGC arbors. The diameters of branches of A, B, C, and D orders in the NMDA-treated arbors remained roughly unchanged compared to the equivalent branch orders in non-NMDA-treated arbors. However, the diameter of $\mathrm{E}$ branches (end branches) of NMDA-treated arbors are apparently larger than that of the $\mathrm{E}$ and $\mathrm{F}$ branches of nonNMDA-treated arbors $(\Delta$ : E vs E, $p=0.001 ; *$ : E vs F, $p<0.001)$. This effect of NMDA treatment on branch diameter is graphically illustrated in the inset. contact of retinal arbors caused by chronic exposure to low levels of NMDA is significant at the $p=0.024$ level (one-way ANOVA).

There are, however, several limitations to the sampling methods used for these estimates. The estimated values are valid for "relative" comparisons across branch orders within a single arbor, or between branch orders across different arbors, but they are not accurate estimates of the "absolute" values for synaptic contact area supported by individual arbors. The measurements are underestimates of the real values for the following reasons. The type IV retinal arbors are generally planar and extend parallel to the tectum's dorsal surface. The tectal sections containing the type IV axons were cut parallel to this surface, and the light microscope reconstructions were made in the same plane. However, the data still contain a small underestimate of the lengths of axonal branches that were not precisely parallel to the tectal surface. A second factor that contributes to an underestimate of synaptic contact is the criteria for a synaptic release site, namely, a pre- and postsynaptic membrane thickenings and the associated synaptic vesicles. This dictates that all HRP-labeled syn-

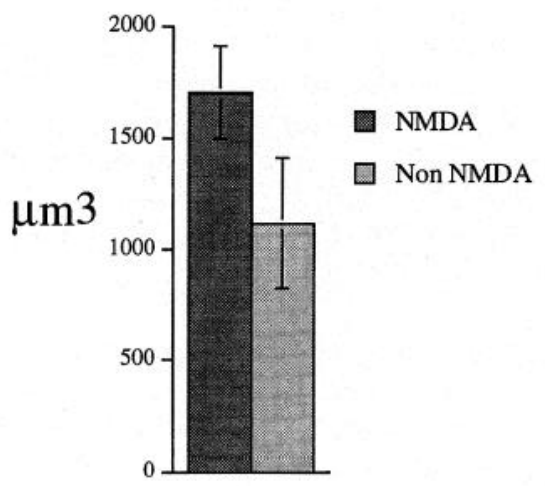

Figure 12. Axonal volume of single arbors. The branch reduction caused by NMDA treatment does not decrease total volume of individual arbors. It actually increases the volume of the axonal tree (one-way ANOVA, $p=0.018$ ). 


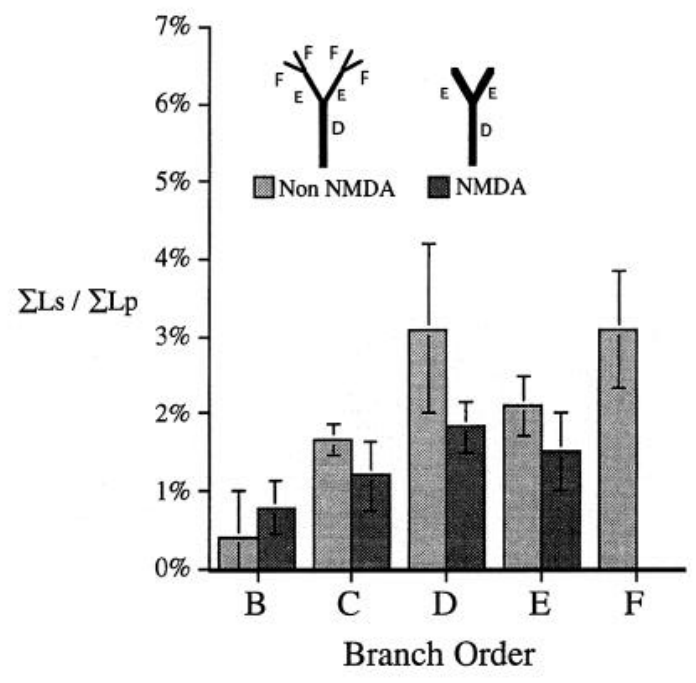

Figure 13. The proportion of axonal membrane in synaptic contact. The $\Sigma L s / \Sigma L p$ values appear to be smaller in more proximal branch orders, and larger in more distal branch orders. Comparisons of the $\Sigma L s /$ $\Sigma L p$ values of NMDA-treated arbors to that of non-NMDA-treated arbors indicate that the $\mathrm{B}, \mathrm{C}, \mathrm{D}, \mathrm{E}$ branches remained roughly unchanged in this parameter (NMDA vs non NMDA. One-way ANOVA: B branches, $p=0.384$; $\mathrm{C}$ branches, $p=0.106$; $\mathrm{D}$ branches, $p=0.065 ; \mathrm{E}$ branches, $p=0.112$ ). However, for the end branches (order $\mathrm{E}$ ) of NMDA-treated arbors, the $\Sigma L s / \Sigma L p$ value is significantly lower than that of the end branches (order F) of non-NMDA-treated arbors ( $E$ vs F branches, $p<0.014$ ).

aptic release sites that were not clearly viewed in cross-section could not be counted as synapses. Therefore, synaptic contact area should be reduced relative to axonal membrane area in the values we have obtained.

\section{Mean synaptic size}

To determine whether the data in Figure 14 reflected a decrease in the size of remaining synapses in NMDA-treated arbors in addition to the loss of synapses due to branch elimination, the mean synaptic size (mean $L s$ ) were compared between the NMDA-treated and the non NMDA-treated arbors. Figure $15 a$ shows that the mean synaptic size does not change with NMDA treatment (nested ANOVA: between group, $p=0.704$; within group, $p=0.033$ ). Since the total synaptic contact area made by NMDA-treated arbors is decreased and the mean synaptic size remains unchanged, it follows that the total "number" of synapses supported by each NMDA-treated arbors is decreased. Thus, it appears that the number of synapses, rather than the sizes of the synapses, is regulated with NMDA treatment. In addition, as can be seen in Figure $15 b$, the size of synapses appeared fairly constant across branch orders. This was true for both NMDA-treated and non-NMDA-treated arbors (ANOVA, $p>0.3$, for all paired comparisons between branch orders within the same treatment group, or between branch orders of different treatment group).

\section{Discussion}

The current analysis indicates that chronic NMDA treatment which prunes the branches of type IV arbors at the light microscope level, significantly reduces the amount of synaptic contact area maintained by these arbors (Fig. 14b). Since the size of these synaptic release sites in these arbors remains unchanged (Fig. 15), the number of synapses must be reduced. In addition, a.
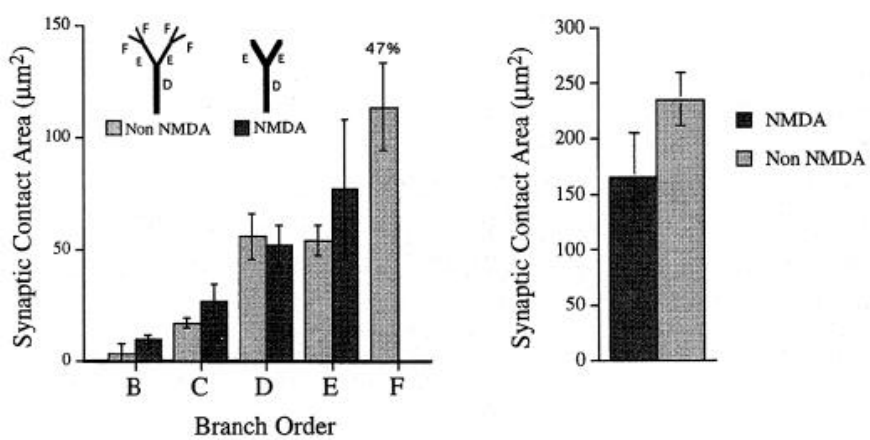

Figure 14. $a$, Synaptic contact area contributed by branches of each order. The end branches (order F) of the non-NMDA-treated arbors contribute almost half of the total synaptic contact of the entire arbors. The NMDA-treated arbors lack the $\mathrm{F}$ branch order. $b$, Comparison of the averaged total synaptic contact area of all NMDA-treated and all non-NMDA-treated arbors. The total synaptic contact area made by each arbor was reduced by NMDA treatment (one-way ANOVA, $p=$ $0.024)$.

the observations also suggest that the parts of the arbors constituting distal branches prior to NMDA treatment, were simply reabsorbed into the next most proximal branches. The most distal $\mathrm{E}$ branches of NMDA-treated arbors are equal in length to the E branches of non NMDA-treated arbors (Fig. 10), but the diameter of $\mathrm{E}$ branches in NMDA-treated arbors is larger than that of the E branch in non NMDA-treated arbors (Fig. 11). This pattern suggests a loss of branches and synapses furthest from the center of the topographically appropriate loci of each arbor.

The results, however, also indicate a possible functional compensation in the NMDA-treated arbors that could result from the increased diameter of the remaining $\mathrm{E}$ branches. Namely, the enlarged diameter should offer less internal resistance to passive current flow into the new most distal branches. This increased electronic spread, compounded by the fact that there are fewer synaptic release sites in the arbor, implies that each of the re- a.

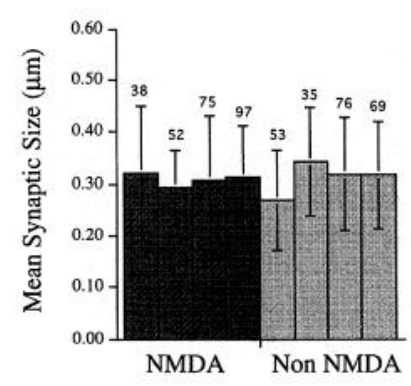

b.

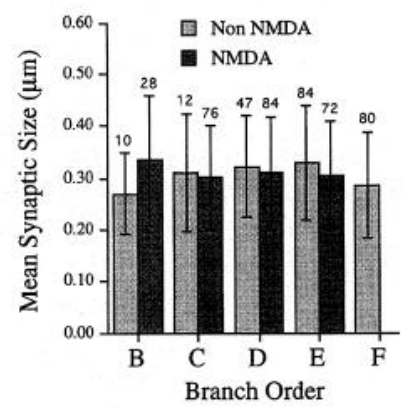

Figure 15. a, Mean synaptic size does not change with NMDA treatment (nested ANOVA, $p=0.7$ ). Each bar represents the mean synaptic size of a single retinal arbor. The value above each bar shows the number of synapses sampled for each retinal arbor. $b$, Mean synaptic size grouped by branch orders. The size of synapses appeared fairly constant across branch orders in both NMDA-treated and non-NMDA-treated arbors (ANOVA, $p>0.3$, for all paired comparisons between branch orders within the same treatment group, or between branch orders of different treatment groups). The value above each bar shows the number of synapses sampled for each branch order. 
maining release sites is likely to have more of the biochemical machinery for synaptic release. Thus the remaining synapses in NMDA-treated arbors are likely to be more effective. In short, it seems likely that, despite the loss of synaptic contact area, NMDA treatment and the subsequent pruning of retinal arbors may result in a focusing and strengthening of retinal synaptic activation in the center of the topographically correct locus. Indeed, our earlier study of this neuropil demonstrated that NMDA treatment alters synaptic release sites so that they look as if they would be more effective in driving postsynaptic targets: the preand postsynaptic densities are thickened, and the synaptic release sites are locally clustered (Yen et al., 1993).

It was necessary to concentrate the current analysis on only one of the several RGC arbor types in the frog retinotectal projection to reduce heterogeneity with our sampling methods. However, the synaptic effects of chronic NMDA treatment in type IV arbors are likely to generalize to all RGC arbor types because the features of the frog retinal projection that are most important for activity-dependent plasticity are shared by all of the RGC arbors. All RGC arbors appear to use glutamate as, at least, one of their transmitters (Langdon and Freeman, 1986; Hickmott and Constantine-Paton, 1993), and all segregate into stripes in doubly innervated tecta (Constantine-Paton and Law, 1978). All RGC arbors shift their arborization site during development (Gaze et al., 1979; Reh and Constantine-Paton, 1984), and are susceptible to pruning upon chronic NMDA treatment (Cline and Constantine-Paton, 1990, Yen et al., 1993).

The current analysis also highlights several unexpected features of both NMDA and non-NMDA-treated arbors that have potentially broad implications when relating light microscope morphology to synaptic release sites. First, despite the fact that large segments of unmyelinated axon are present in the proximal (B) branches of the type IV arbors, most of the synaptic contact is found in the more distal (D, E, F) branches. In fact, the most distal ( $F$ order) branches contribute almost half of the total synaptic contact of the non-NMDA-treated arbors (Fig. 14a). These same branches are the youngest and most mobile regions of the arhor as judged by the occasional presence of growth cones, and by the fact that during this postmetamorphic period the arbors continue to shift toward the central tectum (Gaze et al., 1979; Reh and Constantine-Paton, 1984; Hitchcock and Easter, 1987). Their labile state is also suggested by dynamic imaging of retinal arbor movement in living Xenopus tecta, where the analogous distal branches of these arbors were shown to turn over in a matter of hours (O'Rourke and Fraser, 1990; O'Rourke et al., 1994). Thus, we are led to the suggestion that a significant portion of the retinal synapses (the ones localized on the distal branches) must be very transient structures.

In light of the evidence that the synapses on distal branches must either be very young or about to be withdrawn, it was surprising that the synaptic release sites on these branches did not differ from those on the more proximal branches of the arbor (Fig. 15b). It is possible that the "age" of a synapse in these retinal arbors is not reflected in their size, although this would be in contrast to a number of earlier observations (Kalil et al., 1986; Markus et al., 1987; Buchanan et al., 1989). Alternatively, all the synaptic release sites of these arbors, whether they are located on the distal or proximal branches, could be equally short lived and formed at a fixed size. There have been previous suggestions of this in remodeling mammalian terminals (Markus et al., 1987; I andis et al., 1989).

Another finding of the present analysis, relevant to the issue of NMDA receptor activation and the modulation of process growth, is the increase in diameter of the E branches of NMDAtreated arbors. This increase actually compensates for the volume of axumal arbor that is lost in the F branches. Therefore, there is no net loss of axon volume in the NMDA-treated arbors. A previous study noted a reduction in the neuropil thickness of NMDA-treated tectal lobes (Cline and Constantine-Paton, 1990), and attributed this reduction to a loss of retinal axon volume. If axoplasmic volume cannot account for the reduction in neuropil thickness, then some other components of the neuropil must be reduced by the NMDA treatment. One strong possibility is the postsynaptic dendritic volume. Indeed, numerous studies have implied that activity can regulate the remodeling of postsynaptic dendrites during development (Katz and Constantine-Paton, 1988; Rocha et al., 1991; Bodnarenko and Chalupa, 1993; Kalb, 1994).

In short, we have demonstrated a significant reduction of synaptic contact within single retinal arbors following chronic NMDA treatment, and this reduced synaptic contact appears to be due to the pruning of the end branches of the arbors. These observed changes following NMDA treatment could have at least three different causes that do not require an activity-dependent mechanism involving postsynaptic NMDA receptors. First, chronic NMDA treatment could be having a toxic effect on the developing retinotectal neuropil. However, this appears to be ruled out because the same NMDA treatment did not alter retinal ganglion or tectal cell number (Cline et al., 1987), and we found no signs of increases in degenerative responses such as vacuolated nuclei, phagocytotic processes, or electron-dense profiles in the NMDA-treated tecta (Yen et al., 1933). Second, NMDA could be acting presynaptically, directly on the retinal arbors. This is unlikely because the observed NMDA effects on branch reduction (Cline and Constantine-Paton, 1990), and on synaptic fine structure and clustering (Yen et al., 1933) are absent from similarly treated tecta innervated by only one eye. We think that a significant difference between doubly and singly innervated tecta is that the former has less correlated activity per unit volume of neuropil than the latter (Cline and Constantine-Paton, 1990). The final possibility is that NMDA treatment has a postsynaptic effect mediated through non-NMDA receptors. This can not be eliminated entirely. However, another study using patch clamp recordings on single tectal cells after chronic NMDA treatment has failed to find any effect on non-NMDA ionotropic glutamate receptor in the same cells that show a pronounced downregulation of the NMDA current (Hickmott and Constantine-Paton, 1991, 1993b, unpublished observations). Thus, we believe that the simplest explanation for these data is that the NMDA treatment selectively eliminated poor-correlated inputs by decreasing the effectiveness of NMDA receptors on tectal neurons, and that mainteriance of an axonal branch requires a minimum density of synapses that are correlated with converging neighbors. This is expected if the NMDA receptor is an integral part of the mechanism that initiates the selective stabilization of synapses with correlated activity. With this mechanism, NMDA receptor down regulation should raise the threshold for detecting correlated synapses, and synapses with lower correlations in their activity patterns should be lost together with the branches bearing them.

\section{References}

Bear M, Kleinschmidt A, Gu Q, Singer W (1990) Disruption of experience dependent modifications in striate cortex by infusion of an NMDA receptor antagonist. J Neurosci 10:909-924. 
Bodnarenko SR, Chalupa LM (1993) Stratification of ON and OFF ganglion cell dendrites depends on glutamate-mediated afferent activity on the developing retina. Nature 364:144-146.

Buchanan J, Sun Y-A, Poo M-M (1989) Studies of nerve-muscle interactions in Xenopus cell culture: fine structure of early functional contacts. J Neurosci 9:1540-1544.

Changeux J-P, Danchin A (1976) Selective stabilization of developing synapses as a mechanism for the specification of neural networks. Nature 264:705-712.

Cline HT, Constantine-Paton M (1989) NMDA reccptor antagonists disrupt the retinotectal topographic map. Neuron 3:413-426.

Cline HT, Constantine-Paton M (1990) NMDA receptor drug treatment alters RGC terminal morphology in vivo. J Neurosci 10:1197-1216.

Clíne HT, Debski E, Constantine-Paton M (1987) NMDA receptor antagonist desegregates eye specific strips. Proc Natl Acad Sci USA 84:4342-4345.

Constantine-Paton M, Law MI (1978) Eye-specific termination bands in tecta of three-eyed frogs. Science 202:639-641.

Debski EA, Cline HT, McDonald JW, Constantine-Paton M (1991) Chronic application of NMDA decreases the NMDA sensitivity of the evoked potential in the frog. J Neurosci 11:2947-2957

Gaze RM, Keating MJ, Ostberg A, Chung SH (1979) The relationship between retinal and tectal growth in larval Xenopus. Implication for the development of the retinotectal projection. J Embryol Exp Morphol 53:103-143.

Grüsser O-J, Grüsser-Cornehls U (1964) Functional organization of receptive fields of inovement detecting neurons in the frog's retina. Pfluegers Arch Ges Physiol 279:88-93.

Hahm J-O, Langdon RB, Sur M (1991) Disruption of retinogeniculate afferent segregation by antagonists to NMDA receptors. Nature 351: $568-570$.

Hickmott P, Constantine-Paton M (1991) Quantitative analysis of agonist-evoked currents in identified tectal neurons of Rana pipiens. Proc Soc Neurosci 17:1134.

Hickmott P, Constantine-Paton M (1993a) The contributions of NMDA, non-NMDA, and GABA receptors to postsynaptic responses in neurons of the optic tectum. J Neurosci 13:4339-4353.

Hickmott P, Constantine Paton M (1993b) Effects of chronic N-methyD-aspartate treatment on spontaneous EPCs recorded from Rana pipiens optic tectum. Proc Soc Neurosci 19:452.

Hitchcock P, Easter S (1987) Evidence for centripetally shifting terminals on the tectum of postmetamorphic Rana pipiens. J Comp Neurol 266:556-564.

Kalb R (1994) Regulation of motor neuron dendrite growth by NMDA receptor activation. Development, in press.

Kalil RE, Dubin MW, Grayson S, Stark LA (1986) Elimination of action potentials blocks the structural development of retinogeniculate synapses. Nature 323:156-158.

Katz LC, Constantine-Paton M (1988) Relationships between segregated afferents and postsynaptic neurons in the optic tectum of threeeyed frogs. J Neurosci 8:3160-3180.

Kleinschmidt A, Bear MF, Singer W (1987) Blockade of NMDA re ceptors disrupts experience-dependent plasticity of kitten striate cortex. Science 238:355-358.

Landis DMD, Payne HR, A. WL (1989) Changes in the structure of synaptic junctions during climbing fiber synaptogenesis. Synapse 4:281-293.

Langdon RB, Freeman JA (1986) Antagonists of glutaminergic neu- rotransmission block retinotectal transmission in goldfish. Brain Res 398:169-174.

Law MI, Constantine-Paton M (1981) Anatomy and physiology of experimentally produced striped tecta. J Neurosci 1:741-759.

Markus EJ, Petit TL, Le Boutillier JC (1987) Synaptic structural changes during development and aging. Dev Brain Res 35:239-248.

Maturana HR, Lettvin JY, McCulloch WS, Pitts WH (1960) Anatomy and physiology of vision in the frog (Rana pipiens). J Gen Physiol [Suppl] 43:129-175.

O'Rourke NA, Fraser SE (1990) Dynamic changes in optic fiber terminal arbors lead to retinotopic map formation: an in vivo confocal microscopic study. Neuron 5:159-171.

O'Rourke NA, Cline HT, Fraser SE (1994) Rapid remodeling of retinal arbors in the tectum with and without blockade of synaptic transmission. Neuron 12:921-934

Rabacchi S, Bailly Y, Delhaye-Bouchaud N, Mariani J (1992) Involvement of the NMDA receptor on synapse elimination during cerebellar development. Science 256:1823-1825.

Reh TA, Constantine-Paton M (1984) Retinal ganglion cell terminals change their projection sites during larval development of Rana pipiens. J Neurosci 4:442-457.

Riccio RV, Matthews MA (1985a) Effects of intraocular tetrodotoxin on dendritic spines in the developing rat visual cortex: a Golgi analysis. Dev Brain Res 19:173-182.

Riccio RV, Matthews MA (1985b) The postnatal development of the rat primary visual cortex during optic nerve impulse blockade by intraocular tetrodotoxin: a quantitative electron microscopic analysis. Dev Brain Res 20:50-68.

Rocha M, Ramoa A, Hahm J, Sur M (1991) NMDA antagonist infusion during ON/OFF sublaminar segregation alters dendritic morphology of cells in ferret LGN. Proc Soc Neurosci 17:1136.

Schmidt JT (1990) Long-term potentiation and activity dependent retinotopic sharpening in the regenerating retinotectal projection of goldfish: common sensitive periods and sensitivity to NMDA blockers. J Neurosci 10:233-246.

Shatz CJ (1990) Impulse activity and the patterning of connections during CNS development [review]. Neuron 5:745-756.

Silberstein G, Danicl C (1982) Elvax 40P implants: sustained, local release of bioactive molecules influencing mammary ductal development. Dev Biol 93:272-278.

Simon DK, Prusky GT, O'Leary DD, Constantine-Paton M (1992) $\mathrm{N}$ methyl-D-aspartate receptor antagonists disrupt the formation of a mammalian neural map. Proc Natl Acad Sci USA 89:10593-10597.

Stent GS (1973) A physiological mechanism for Hebb's postulate of learning. Proc Natl Acad Sci USA 70:997-1001

Stirling RV, Merrill EG (1987) Functional morphology of frog retinal ganglion cells and their central projections: the dimming detectors. $J$ Comp Neurol 258:477-495.

Strahler AN (1952) Hypsometric analysis of erosional topograph. Bull Geol Soc Am 63:1171-1182.

Taylor AC, Kollros JJ (1946) Stages in the normal development of Rana pipiens larvae. Anat Rec 94:7-23.

Udin SB, Scherer WJ (1990) Restoration of the plasticity of binocular maps by NMDA after the critical period in Xenopus. Science 249: 669-672.

Yen L-H, Sibley JT, Constantine-Paton M (1993) Fine-structural alterations and clustering of developing synapses after chronic treatments with low levels of NMDA. J Neurosci 13:4949-4960. 